\title{
Gestión por competencias y su relación con el clima laboral en una empresa de servicios, consultoría y outsourcing, Lima, Perú
}

Recibido: 27/03/2020 Aceptado: 11/08/2020 Publicado: 31/12/2020

Daniel Enrique Rey de Castro Hidalgo ${ }^{1}$ JAIME EDUARDO GUTIÉRREZ ASCÓN ${ }^{2}$ JUSTINA URIBE KAJAT ${ }^{3}$

\begin{abstract}
RESUMEN
El objetivo principal fue determinar el nivel de relación entre un modelo de gestión de competencias población fue de 350 trabajadores de una empresa peruana con muestra ajustada de 120 al $95 \%$ de nivel de confianza. Los métodos de análisis se realizaron a través de encuestas con dos tipos de escala, de tipo Likert y dicotómica. Según los resultados la variable Gestión por Competencias y el Clima Laboral se correlacionan significativamente y en forma positiva (0.622). Las dimensiones de la variable Gestión por Competencias son: Planeamiento y Difusión del Plan Estratégico, Evaluación de Competencias, Formación Basada en Competencias y la variable independiente Clima Laboral. Se concluye que la Gestión por Competencias y todas sus dimensiones se relacionan con el Clima Laboral.
\end{abstract}

Palabras clave: Gestión por Competencias; Planeamiento y Difusión del Plan Estratégico; Clima Laboral.

MANAGEMENT BY COMPETENCIES AND ITS RELATIONSHIP WITH THE WORK ENVIRONMENT IN A SERVICES, CONSULTING AND OUTSOURCING Company, Lima, Peru

\section{ABSTRACT}

The main objective was to determine the level of relationship between a skills management model and the work environment of workers in a service, consulting and outsourcing company. The population was 350 workers from a Peruvian company with an adjusted sample of 120 to $95 \%$ confidence level. The analysis methods are carried out through surveys with two types of scale, Likert type and dichotomous. According to the results of the variable management by competences and the work environment, the difficulties and the positive form are correlated $(0.622)$. The dimensions of variable management by competences are: planning and dissemination of the strategic plan, identification of competencies, basic training in competencies and work environment. An application scheme of a competency management model is proposed. It is concluded that competency management and all its dimensions are related to the work environment Key words: Competences, strategic plan, leadership, work environment.

Keywords: Management by competences; strategic planning; work environment.

\section{INTRODUCCIÓN}

La reflexión sobre la Gestión por Competencias y su respectiva relación con el Clima Laboral es un tema que exige un análisis desde distintas perspectivas en el campo del conocimiento y de las actividades laborales.

Con el paso del tiempo, el avance de la tecnología y la consecuente globalización, las organizaciones se vieron obligadas a evolucionar para subsistir, mantenerse o sobre todo, posicionarse en un puesto de liderazgo. En esta medida las estructuras organizacionales rígidas y burocratizadas se han transformado (o lo vienen haciendo) en estructuras cada vez flexibles, es decir orgánicas.

Durante este proceso de evolución es que aparece la concepción de competencias y por ende su gestión. Lawler (1994) estableció como una de las ventajas de pasar a una empresa gestionada por competencias, el paso de la burocratización a un modelo sistémico - orgánico.

Muchos autores han definido el término competencia. El primero a quién se le atribuye su definición es a McClelland (1973), que indicó que "El rendimiento de una persona no dependía únicamente de los títulos que poseía ni de sus resultados en tests de inteligencia, sino que dependía más de características propias y competencias" (p. 6). Posteriormente, Boyatzis (1982) las definió como "Características subyacentes en una persona que están causalmente relacionadas con una actuación de éxito en un puesto de trabajo" (p. 23).

Mertens (2000) mencionó competencia como: "La capacidad demostrada por una persona para lograr un resultado, que puede $o$ no convertirse en un avance efectivo" (pág. 16).

1 Ingeniero industrial. Consultor independiente ORCID: https://orcid.org/0000-0002-0610-2150 E-mail: danielreydecastro@gmail.com

2 Ingeniero Industrial. Docente. Universidad Nacional José Faustino Sánchez Carrión, Huacho. ORCID: https://orcid.org/0000-0003-4065-3359 E-mail: ing jagu@hotmail.com

3 Doctora en administración y doctora en educación. Docente. Universidad Nacional Federico Villarreal.

ORCID: https://orcid.org/0000-0002-2691-3073

E-mail: juribe@unmsm.edu.pe 
Navío (2002) propuso la competencia como:

Conjunto de elementos combinados (Conocimientos, habilidades, actitudes, valores, normas, etc.) que se integran sobre la base de una serie de atributos personales (capacidades, motivos, rasgos de personalidad, aptitudes, etc.), tomando como referencia las experiencias personales y profesionales y manifestándose mediante determinados comportamientos o conductas en el contexto de trabajo (p. 2).

En el mismo sentido, Alles (2006) indicó que las competencias son "Características de personalidad devenidas comportamientos que generan un desempeño exitoso en un puesto de trabajo" (p. 163).

De estas definiciones se puede sintetizar que competencias son un conjunto de características necesarias que debe tener un trabajador para cumplir con eficacia el papel que le corresponde en su organización y las cuáles pueden ser medibles en tiempo y espacio.

En esta investigación, se plantea encontrar la relación entre la variable independiente Gestión por Competencias (X) compuesta de cuatro dimensiones propuestas: Planeamiento y difusión del plan estratégico $\left(X_{1}\right)$, Identificación de Competencias $\left(X_{2}\right)$, Formación Basada en Competencias $\left(X_{3}\right)$ y Evaluación de Competencias $\left(\mathrm{X}_{4}\right)$ con la variable dependiente Clima Laboral $(\mathrm{Y})$ y sus dimensiones más importantes según la problemática de la organización estudiada.

Se entiende la Gestión por Competencias como un grupo de teorías y procedimientos desarrollados en conjunto cuyo objetivo es potenciar las acciones de las personas para maximizar su desempeño dentro de la organización. Por esta razón, la Gestión por Competencias debe encontrarse en un marco que permita su diseño, desarrollo y efectiva implementación. Además, ella se define como la estructuración de la organización con base en el factor humano de modo que permita aprovechar las capacidades de los colaboradores y desarrollarlas (Ernst \& Young, 1998, p. 5).

En las empresas, donde se busca mejorar el desempeño organizacional, generalmente se prioriza la gestión del conocimiento. Sin embargo, el planeamiento estratégico es el que contribuye a la gestión del conocimiento en la mejora del desem- peño organizacional. Así mismo, es importante la participación de los trabajadores en el proceso de planeamiento. Es así, que el estudio de Noermijati, Achmad y Grace (2019), concluye que "Knowledge management has indirect effect on organizational performance. It is necessary for good planning strategic. By doing innovation, sharing opinions, employee's involvement in decision making process, it creates better strategic planning, which in turn will affect on organizational performance" (p.96).

Una relación estadística importante del planeamiento estratégico en una organización fue investigada por Aziz(2019) "The results showed that the strategic planning has a good statistically significant impact on financial, customer, and learning and growth. The results also showed a medium statistically significant impact of strategic planning on internal business process." (p.140).

De lo anterior, se deduce que los miembros de la organización deben poseer una noción clara de la visión, misión, objetivos, valores y demás sub elementos para que resulte idóneo iniciar el proceso de Identificación de Competencias. Así lo afirma Alles (2011) al señalar que "A partir de la Misión y Visión se definirá, junto con la máxima conducción, cuáles son las características básicas requeridas para los distintos puestos y los valores a seguir" (p.26). La importancia de llevar a cabo la Identificación de Competencias de manera exhaustiva y consciente por parte de la organización permite tener una visión amplia y optimizar los recursos para la etapa de formación. Dicha importancia se pone de manifiesto en la investigación de Bonal-Ruiz et al. (2017), afirmando que:

Hubo un predominio en la concepción educativa de la promoción de salud más que de los aspectos ambientales, comunitarios e intersectoriales de ésta. Se identificaron conocimientos, actitudes y habilidades resaltando la necesidad de más habilidades y actitudes que de conocimientos, estas últimas se enfocaron en la implementación de modelos y teorías actualizadas (p.32)

En el campo de la educación, al identificar los perfiles de las competencias adquiridas por estudiantes, Giménez \& Aymerich (2016) encontraron que "Las competencias $1,7,8,9,10,11$ y 12 se aprendieron haciendo, pensando y escribiendo mediante modelos (X3) que corresponden a diferentes tipos de conocimientos o a su combinación" (p.83). Donde los "affordances" de los instrumentos de medida, 
la interpretación de fenómenos, la redacción de un procedimiento, la justificación de operaciones de un procedimiento, la técnica utilizada para cada propiedad y elección de los parámetros ensayados, la justificación de la fórmula de cálculo y finalmente la valoración del resultado, fueron aprendidos como consecuencia de actividades de acción, de pensamiento y de escritura en estudiantes; entonces, se puede afirmar que el modelo de competencia aporta posibilidades reales de transformación profunda no solo para la empresa sino para la persona, hecho que permite la involucración de los hombres en su puesto de trabajo y organización.

Así mismo, los constantes cambios en el mercado laboral exigen actualizaciones y nuevos métodos en el proceso de Identificación de Competencias requeridas para un determinado puesto de trabajo (Lula, Oczkowska \& Wiśniewska, 2019). En este sentido, otros autores proponen diseñar esquemas de competencias siguiendo patrones existentes en los mercados laborales. De cualquier modo, el objetivo resulta siendo el de diseñar un plan de Formación Basada en Competencias para potenciar aquellas necesarias para el trabajador y la organización, tal como lo indica Lula et al. (2019):

The results showed in the paper show that the concept of competency schema can be used for representation of crucial patterns existing on labour markets. Schemas may give a detailed insight into requirements of employers and can be helpful for estimating competency gap or design study or training programs appropriate for the needs of the labour (p.16).

En el ámbito de la Formación Basada en Competencias surgen diversas posturas respecto a cuáles o qué tipo de competencias deben ser desarrolladas. De hecho, desde la década de 1980-1990 las prácticas de los modelos de gestión de recursos humanos se han implementado para mejorar los niveles de conocimientos, actitudes y habilidades del personal en las organizaciones; donde las competencias individuales derivan de las habilidades de la organización; por lo tanto, el "Modelo de gestión de competencias se presenta como un sistema que está menos conectado con las actividades regulares y operativas que los sistemas basados en la posición." (Rocha, Passador \& Shinyashiki, 2017, p.236). En este sentido, autores señalan la importancia en la formación en ciertas competencias basadas en la necesidad del mercado. Al respecto, Rivero, Martín \& Gil (2015) apuntan que "La formación en la competencia de resolución de problemas es ampliamente demandada por el ámbito laboral" (p.50). Además, se defiende mucho el uso de simuladores como el proceso lúdico "serius games" (SG) que se aplican en este reto, utilizando cuestionarios pre y post actividad. A respecto, señalan Calabor, Mora \& Moya (2018) que "Los estudiantes perciben que el SG contribuye significativamente a la adquisición de las competencias. Observamos también de manera directa que se fomenta el trabajo en equipo" (p.38). Se espera, por tanto, que las organizaciones elaboren procesos de Formación Basado en Competencias realizando un análisis minucioso de aquellas competencias necesarias para mejorar el rendimiento de un trabajador en su respectivo puesto de trabajo.

Hasta aquí surgen las preguntas: ¿Los resultados serán los esperados? ¿Cómo se van a medir y después de cuánto tiempo? Evidentemente, será necesario responder a estas preguntas y diseñar métricas adecuadas que nos permita un feedback a través de una Evaluación de Competencias. De esta forma, se obtendrá la certeza de un desarrollo óptimo en el proceso formativo (Fernandez, 2006). Sin embargo, es importante no confundir Evaluación de Competencias con evaluación por desempeño, lo que podría desviar el foco de lo que se necesita evaluar y además, generar una percepción negativa en el trabajador evaluado. En este sentido Spencer \& Spencer (1993) brindan una serie de inconvenientes de una evaluación por desempeño tradicional.

Se comprende como Clima Laboral al ambiente socio-técnico que comparte la población laboral para desarrollar su trabajo; donde, la calidad del clima, está justificado por la satisfacción laboral, hecho que contribuye a mejorar y sostener los indicadores productivos como rendimiento y atención en las organizaciones. Para evaluar el nivel de desempeño laboral y comercial de las empresas, es conveniente medir el nivel de liderazgo que se relaciona con los niveles de confianza interpersonal para mejorar los procesos de entrega del servicio o producto, donde se encuentra que otorgar incentivos emocionales es más efectivo que la sanción punitiva, tal como lo afirman en su estudio Strukan \& Nikolić (2019) "Leaders need to lower their performance expectations, reduce punishment, improve their relationship with employees, increase rewards in line with opportunities, and refine their strategic knowledge, thinking, and action" (p. 48). En la búsqueda de encontrar nuevos estados de conocimientos al explorar las interacciones interpersonales clave; al respecto, Atkins (2019) encontró que "The development of trusting relationships, and sufficient 
levels of risk-taker, self-confidence to sustain engagement in the generativity processes, as change cycles" (p.48), demostrando que al desarrollar los niveles de confianza interpersonales entre todos en la empresa se mejora la toma de decisiones para enfrentar el riesgo y los procesos de generación de iniciativas como un proceso de cambio.

Sin embargo, es el compromiso personal y corporativo como componente esencial en la cultura organizacional que sostiene y mejora el Clima Laboral en las organizaciones e indica que existen consecuencias de mejor servicio en la medida que se alcanzan mejores resultados en los aprendizajes basados especialmente en la confianza personal y empoderamiento. Sotelo (2018) resalta la importancia del compromiso en su investigación al señalar que "El compromiso organizacional, determinará el nivel de Identificación de Competencias que el empleado tiene hacia su organización desde sus componentes afectivos, de continuidad y normativos" (p.45).

Para comprender el liderazgo en las organizaciones, es conveniente formar aquellos facilitadores emergentes líderes del desarrollo organizacional combinando habilidades tanto cognitivas como habilidades emocionales y sinérgicas que se aprenden desde los centros de estudios aplicando métodos interactivos de educación y de capacitación respectivamente, al respecto, Baungaard \& Sanne (2016) en su estudio afirman que es "The university staff and mentors in public and private organisations to adopt interactive methods for education and training" (p.1), señalando también que "The skills needed to be facilitating leaders are, as exemplified above, a combination of cognitive, emotional and synergistic skills" (p.1). Finalmente señalan que las habilidades de liderazgo son aplicables a toda institucion cualquiera sea su naturaleza concluyendo que "The interactive methods used in the course have been successfully applied within companies of various sizes, public institutions, employer organizations, trade unions, virtual networks and non- governmental organizations" (p.20).

Resulta pues evidente, que las dimensiones del Clima Laboral pueden variar de acuerdo a la situación particular de la entidad. Es así, que Da Silva, Castro, Dos Santos \& Neto (2018) en su investigación señalan que se "Suggest the use of the other dimensions of satisfaction and the totalization of the items of the culture scale in new studies in order to verify if new relationships are identified between these constructs" (p. 414). Sin embargo, dada la naturaleza y en base a la teoría las consideramos aplicables a cualquier organización.
Es necesario señalar que, al tratar la Gestión por Competencias, podría resultar obvio el impacto en una de las variables más importantes de toda organización, el Clima Laboral. Sin embargo, resulta importante determinar en qué medida es que se da esta relación, proponiendo un elemento de discusión y aporte para la organización en su interés de mantener a sus trabajadores en armonía.

Para realizar el contraste de variables se planteó la hipótesis principal: La Gestión por Competencias se relaciona con en el Clima Laboral de los trabajadores en una empresa de servicios de consultoría y outsourcing, Lima 2017. Se encontró una relación alta positiva de 0.622 .

Finalmente, se debe mencionar que el resultado de esta investigación sirve como base para que una propuesta de Gestión por Competencias adquiera un argumento más sólido en la implementación para una compañía, no sólo del sector, sino también para cualquier organización en general. La investigación, por tanto, pretende ser un aporte para el desarrollo de las Ciencias Administrativas, al cubrir un campo poco estudiado de manera científica.

\section{MATERIAL Y MÉTODOS}

Investigación de tipo cuantitativa y aplicada, por su nivel es descriptiva donde se miden ciertos aspectos de la variable de estudio y por el diseño es investigación de campo no experimental con aplicación de encuestas. La variable de investigación Gestión por Competencias está construida por las dimensiones Planeamiento y Difusión del Plan Estratégico, Identificación de Competencias, Formación Basada en Competencias y Evaluación de Competencias. La población de sujetos es $\mathrm{N}=350$ trabajadores y se aplicó la ecuación no $=\left(\boldsymbol{Z}^{2} \cdot \boldsymbol{p} \cdot \boldsymbol{q} \cdot \boldsymbol{N}\right)$ / $\left(\boldsymbol{E}^{2}(\boldsymbol{N}-\mathbf{1})+\boldsymbol{Z}^{2} \cdot \boldsymbol{p} \cdot \boldsymbol{q}\right)$ para $\mathrm{n}_{\mathrm{o}}=183$ y con tamaño de muestra ajustado con la ecuación $n=n_{0} / 1+\left(n_{0} / N\right)$ resulta $n=120$ muestreo realizado al $95 \%$ de nivel de confianza.

Para la recolección de datos de la variable independiente Gestión por Competencias se elaboró un cuestionario con una escala tipo mixta. La escala dicotómica Si/No y la escala de Likert con un puntaje mínimo de 1 y máximo de 5 . Para el caso de la variable dependiente Clima Laboral se realizó inicialmente una entrevista abierta a un grupo de 20 trabajadores escogidos al azar para determinar las variables de mayor impacto en el Clima Laboral. Se determinaron cinco, relaciones interpersonales, compromiso, liderazgo y motivación. Con esta información, se diseñó un cuestionario con una escala de Likert con puntaje mínimo de 1 y máximo 
de 5. Las encuestas se realizaron en dos etapas y en el centro de trabajo, durante dos días de la semana. Para determinar la validez de las encuestas se sometieron al juicio experto de cuatro especialistas en el rubro de recursos humanos. La confiabilidad interna del instrumento se midió con Alfa de Cronbach, para la Gestión por Competencias $\alpha=$ $81.40 \%$ y para la variable Clima Laboral $\alpha=87.90 \%$ de fiabilidad interna.

\section{RESULTADOS}

Los resultados descriptivos se muestran a continuación:

En la Figura 1 se observa la distribución de las dimensiones $\mathrm{X}_{1}, \mathrm{X}_{2}$ y $\mathrm{X}_{4}$ de la variable independiente Gestión por Competencias (X).

Se observa en la dimensión Planeamiento y Difusión del Plan Estratégcio $\left(X_{1}\right)$ que el $50 \%$ tuvo una percepción de "Poco", un 42.5\% de "Muy poco" y un $0.8 \%$ de "Nada". Es decir, El 93.3\% evidenció una percepción negativa respecto al Planeamiento y Difusión del Plan estratégico en la organización (Ver fig. 1).Respecto a la dimensión Identificación de Competencias (X2), el 50\% tuvo una percepción de "Poco", el 32.5\% "Muy Poco" y el $4.2 \%$ "Nada". Solo el $13.4 \%$ de los trabajadores encuestados evidenció una percepción positiva, entre "Normal y Bastante".
La dimensión Formación Basada en Competencias (X3) se diseñó para la investigación de tipo dicotómica, razón por la cual muestra los siguientes resultados (Ver figura 2): El 59\% de los trabajadores señaló no haber recibido formación alguna mientras que el $41 \%$ que consideraron haber recibido algún tipo de formación. El $27 \%$ recibió cursos o seminarios orientados a mejorar sus competencias, $11 \%$ fueron rotados además de haber recibido cursos o seminarios. Solo el 3\% indicó haber recibido coaching, fueron rotados y llevaron cursos o seminarios.

Finalmente, la cuarta dimensión Evaluación por competencias (X4), el $58.2 \%$ tuvo una percepción de "Poco", un 30\% de "Muy poco" y un $0.8 \%$ de "Nada". Es decir, El 93.3\% evidenció una percepción negativa respecto al Planeamiento y Difusión del Plan estratégico en la organización (Ver fig. 1).

En la figura 3, se observa que el $45 \%$ de los encuestados se encuentran "En desacuerdo" con el clima laboral. El 37.5\% estar "Medianamente de acuerdo" con el clima laboral, mientras que el $17.5 \%$ está "De acuerdo" con el clima laboral.

\section{Prueba de hipótesis}

La Gestión por Competencias se relaciona con en el Clima Laboral de los trabajadores en una empresa de servicios de consultoría y outsourcing, Lima 2017.



Figura 1. Gráfico de percepción de los trabajadores de dimensiones $X_{1}, X_{2}$ y $X_{4}$ de la variable Gestión por Competencias.

Fuente: Elaboración propia obtenida de cuestionario aplicado a la muestra, año 2017. 


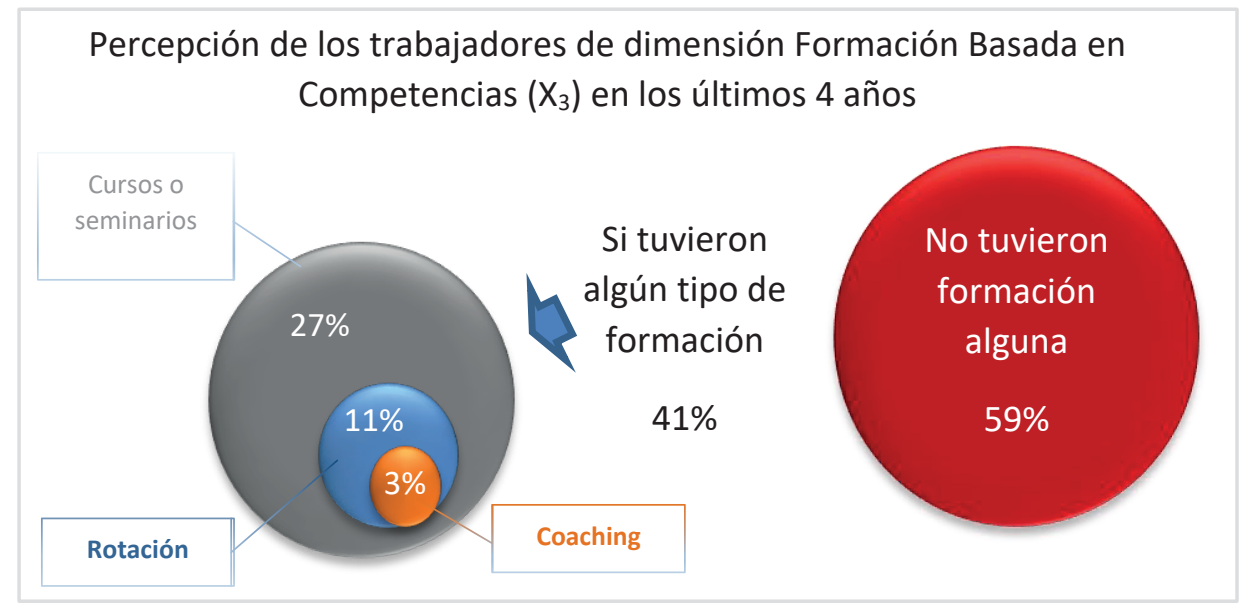

Figura 2. Gráfico de percepción de los trabajadores de dimensión Formación Basada en Competencias de la variable Gestión por Competencias.

Fuente: Elaboración propia obtenida de cuestionario aplicado a la muestra, año 2017.

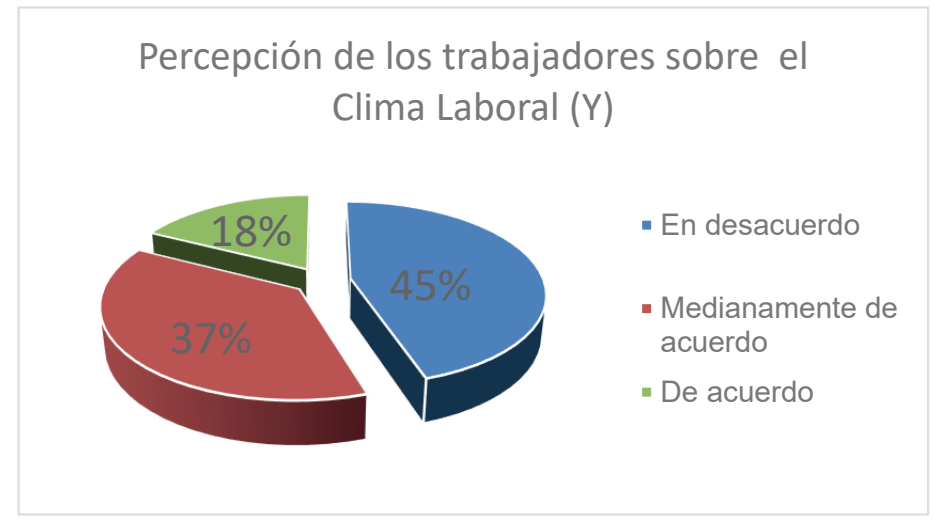

Figura 3. Gráfico de percepción de los trabajadores sobre la variable independiente Clima Laboral.

Fuente: Elaboración propia obtenida de cuestionario aplicado a la muestra, año 2017.

\section{Hipótesis}

La prueba de la hipótesis general de las variables Gestión por Competencias y Clima Laboral presenta el resultado siguiente:

Ho: $r=0$, No existe relación significativa entre la Gestión por Competencias y el Clima Laboral.

$H 1: r \neq 0$, Existe relación significativa entre la Gestión por Competencias y el Clima Laboral.

En la tabla 1 se observa que existe una correlación positiva alta de 0.622 con un nivel de significancia de 0.001 entre la Gestión por Competencias $(X)$ y el Clima Laboral (Y) de los trabajadores en una empresa de servicios de consultoría y outsourcing.
Tabla 1. Valores Rho de Spearman para Gestión por Competencias y Clima Laboral

\begin{tabular}{|l|c|}
\hline & Valor \\
\hline Coeficiente de correlación & 0.622 \\
\hline $\mathrm{N}$ & 120 \\
\hline Sig. (bilateral) & 0.000 \\
\hline
\end{tabular}

Fuente: Elaboración propia obtenido de resultados de software SPSS V25.

El resultado de las correlaciones de las dimensiones X1, X2 y X4 sometidas a la prueba de hipótesis específicas se muestra en la figura 4 . Se observa que existe una correlación positiva alta de 0.501 entre el Planeamiento y Difusión del Plan Estratégico (X1) y el Clima laboral $(Y)$ de los trabajadores en 


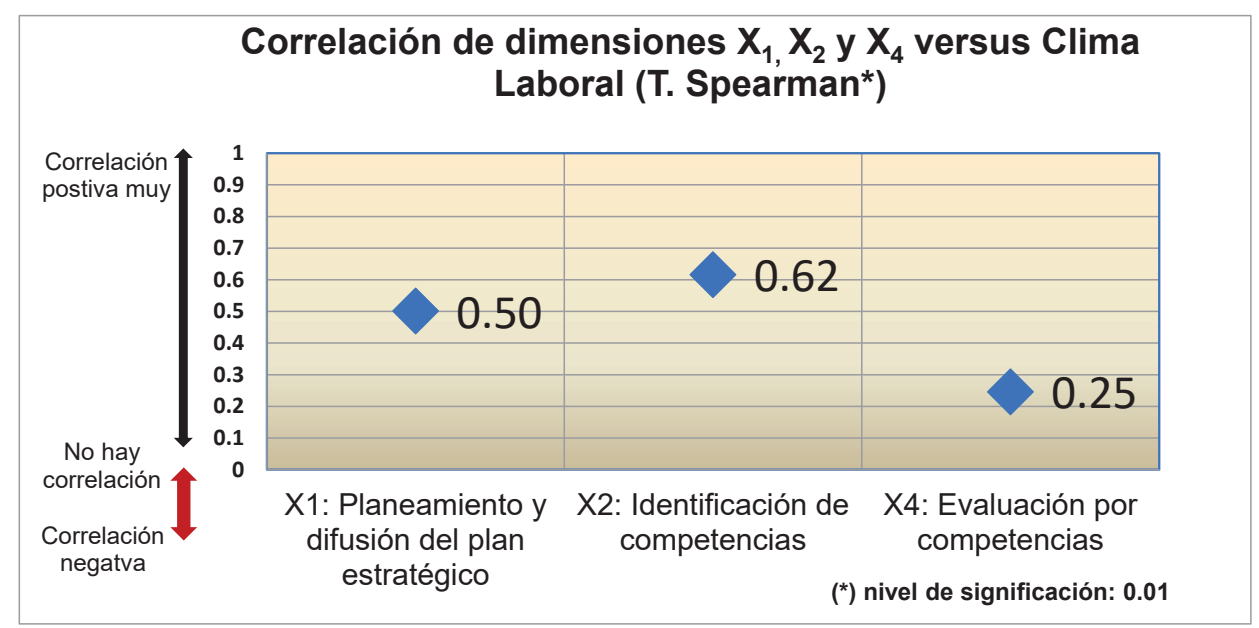

Figura 4. Gráfico de correlación de dimensiones X1, X2 y X4 de la variable Gestión por Competencias versus Clima Laboral.

Fuente: Elaboración propia obtenida de resultados de software SPSS V25.

una empresa de servicios de consultoría y outsourcing. Así mismo, existe una correlación positiva alta de 0.616 entre la Identificación de Competencias (X2) y el Clima Laboral (Y). Respecto a la dimensión Evaluación por Competencias (X4) existe una correlación positiva moderada de 0.246 con un nivel con el clima laboral ( $Y$ ). Las tres pruebas tienen un nivel de significación de 0.01

Tabla 2. Valores Chi-cuadrado para dimensión Formación Basada en Competencias $\left(X_{3}\right)$ y Clima Laboral (Y)

\begin{tabular}{|l|l|l|l|}
\hline \multicolumn{1}{|c|}{ Variables } & $\begin{array}{c}\text { Valor Chi } \\
\text { cuadrado }\end{array}$ & \multicolumn{1}{c|}{ GI } & $\begin{array}{c}\text { Significancia } \\
\text { asintótica }\end{array}$ \\
\hline X3.1 & 58,595 & 2 & 0.000 \\
\hline X3.2 & 19,507 & 2 & 0.000 \\
\hline X3.3 & 26,278 & 2 & 0.000 \\
\hline
\end{tabular}

Fuente: Elaboración propia obtenido de resultados de software SPSS V25.

Según los valores de cada reactivo en la tabla 2 sometidos a la prueba de correlación Chi-cuadrado, existe evidencia estadística para afirmar una relación significativa entre las variables de Formación Basada en Competencias $\left(\mathrm{X}_{3}\right)$ y el Clima Laboral (Y) de los trabajadores en una empresa de servicios de consultoría y outsourcing con un nivel de significación de 0.01 .

\section{Discusión}

Los resultados de la investigación demuestran que la gestión por competencias y el clima laboral se relacionan significativamente en una empresa de servicios, consultoría y outsoursing, Lima 2017, la mismas que fueron validadas mediante las pruebas de correlación T. Spearmann y Chi-cuadrado para probar la relación de las variables.

Esta relación directa la confirma Olaz (2009) en su investigación "Definición de un modelo de clima laboral basado en la gestión por competencias", quien concluyó:

La definición de un modelo de gestión por competencias ayuda a la construcción de una dinámica organizativa y de gestión de recursos humanos, explicitando los valores, los códigos éticos y deontológicos de quien se encuentra en la organización, dirigiendo sus comportamientos, remarcando lo que se espera de él y del grupo, lo que sin duda contribuye notablemente a la búsqueda de un clima laboral satisfactorio (p. 193).

En la misma línea, Moreno, Pelayo y Vargas (2004) señalaron que la implementación de un modelo de gestión por competencias, al permitir gestionar los recursos humanos con mayor análisis, mejora el clima laboral.

La relación significativa entre la Planificación y Difusión del Plan Estratégico y el Clima Laboral, resalta la importancia de la primera dimensión. En este sentido, Alles (2011), señaló la necesidad de redefinir los elementos del plan estratégico frente a la problemática de su vigencia, representatividad y/o poca difusión.

La Identificación de Competencias y su relación con el Clima Laboral presenta el mayor índice de correlación. Por tal motivo, resulta necesario identificar 
aquellas competencias para cada perfil alineados con el plan estratégico. En este sentido, su comunicación transparente y eficiente asegura en el trabajador un norte en su posición en la organización.

Debido a la relación significativa entre la Formación de Competencias y el Clima Laboral, el proceso de formación debe estar además de bien definidas por la organización, coherente con las necesidades del trabajador. Así, Capuano (2004) señaló que "la formación orientada a generar competencia con referentes claros en normas existentes tendrá mucha más eficiencia e impacto que aquella desvinculada de las necesidades de los trabajadores" (p. 149).

La relación moderada entre la Evaluación de Competencias y el Clima laboral en esta investigación se podría deber a la resistencia natural del trabajador a ser evaluado, sobre todo cuando no existe un plan de evaluación adecuada tal como indicó Capuano (2004) "La evaluación de desempeño por competencias constituye el proceso por el cual se estima el rendimiento global del empleado, poniendo énfasis en que cada persona no es competente para todas las tareas y no está igualmente interesada en todas las clases de tareas" (p. 143). Al respecto,

No gestionar una evaluación adecuada le quita la oportunidad a la organización no sólo de hacer un seguimiento adecuado a sus trabajadores, sino también a través de la retroalimentación, de motivar en la mayoría de los casos a qué mejoren el cumplimiento de sus actividades.

Por otro lado, es importante señalar que el clima laboral en una organización se ve afectado por muchos otros factores en menor o mayor medida. Sin embargo, la importancia de una implementación adecuada de gestión por competencias pasa por su alcance, tal como lo afirma García (2010) señalando su implicancia en la integración de los elementos intangibles exclusivos de una organización y el conocimiento superior de las potencialidades de sus trabajadores. Así mismo, el desarrollo de estas competencias, puede significar un incremento en la productividad, demostrado por Burkle \& Day (1986) en su investigación.

El resultado de este tipo de investigación provee a la alta gerencia una herramienta de decisión frente a la implementación de una adecuada gestión por competencias en un escenario en el cual el clima laboral no es el esperado por la organización. Por lo tanto, y a raíz de los resultados, no resulta excluyente que aplicar una gestión eficiente mejore los indicadores en un clima laboral positivo.

\section{CONCLUSIONES}

1. La investigación demuestra que la Gestión por Competencias y el Clima Laboral se relacionan significativamente. Concluyendo que existe una relación positiva y alta de 0.622 .

2. La relación entre la dimensión Planeamiento y Difusión del Plan Estratégico con el Clima Laboral es positiva con un valor de 0.501. En el análisis descriptivo se observa que la percepción general de los trabajadores respecto al Planeamiento y Difusión del Plan Estratégico es negativa. El $42.5 \%$ de los trabajadores considera tener "Poco" conocimiento del Planeamiento y Difusión del Plan Estratégico en su organización.

3. La Evaluación de Competencias se relaciona en 0.616 con el Clima Laboral. El análisis descriptivo de la dimensión Evaluación de Competencias, arroja que en general, los trabajadores manifestaron una percepción negativa.

4. La Formación Basada en Competencias se relaciona significativamente con el Clima Laboral. El análisis descriptivo indicó que en general los trabajadores, según su percepción no recibieron alguna Formación Basada en Competencias. Así mismo, sólo el $11 \%$ participó en algún programa de rotación y en algún curso, seminario o similar para desarrollar alguna competencia.

5. La Evaluación de Competencias se relaciona positiva y moderadamente en 0.246 con el Clima Laboral. En el análisis descriptivo se encontró que la percepción general de los trabajadores es negativa con un $89.2 \%$ de trabajadores que manifestaron tener en su organización una valoración entre "Poco" y "Muy poco" respecto a si esta se basó en una Evaluación de Competencias.

\section{REFERENCIAS BIBLIOGRÁFICAS}

[1] Alles, M. (2006). Selección por competencias. Buenos Aires: Granica.

[2] Alles, M. (2011). Dirección estratégica de recursos humanos. Gestión por Competencias. Buenos Aires: Gránica S.A.

[3] Atkins, S. (2019). Trust-based mentoring towards a new knowledge state as a change cycle: Exploring key interpersonal interactions. International Journal of Evidence Based 
Coaching and Mentoring, 17(2), 36-51. https:// doi.org/10.24384/g11c-ng05

[4] Aziz A. Abdul Rahman, A. (2019). The impact of strategic planning on enhancing the strategic performance of banks: evidence from Bahrain. Banks and Bank Systems, 14(2), 140-151. https://doi.org/10.21511/bbs.14(2).2019.12

[5] Baungaard, L., \& Sanne, M. (2016). Learning facilitating leadership. Studies in Higher Education, 43(8). https://doi.org/10.1080/0307 5079.2016.1266612

[6] Bonal-Ruiz, R., Marzán-Delis, M., \& GarbeyAcosta, A. (2017). Evaluación de Competencias en promoción de salud en médicos de familia de Cuba Estudio cualitativo. Atención Familiar, 24(1), 32-39. https://doi.org/10.1016/j. af.2016.11.002

[7] Boyatzis, R. (1982). The competent manager: A model for effective performance. Nueva york: Jhon Wiley \& Sons.

[8] Burke, M., \& Day, R. (1986). A Cumulative Study of the Effectiveness of Managerial Training. Journal of Applied Psychology, 71, 232 - 245.

[9] Capuano, A. (2004). Evaluación de desempeño: desempeño por competencias. Invenio, 139150.

[10] Calabor, M. S., Mora, A., \& Moya, S. (2018). Acquisition of competencies with serious games in the accounting field: an empirical analysis. Revista de Contabilidad-Spanish Accounting Review, 21(1), 38-47. https://doi. org/10.1016/j.rcsar.2016.11.001

[11] da Silva, L. P., Castro, M. A. R., dos-Santos, M. G., \& Neto, P. J. de L. (2018). Comprometimento no trabalho e sua relação com a cultura organizacional mediada pela satisfação. Revista Brasileira de Gestao de Negocios, 20(3), 401-420. https://doi.org/10.7819/rbgn. v20i3.3947

[12] Ernst \& Young. (1998). Manual del director de recursos humanos. Madrid: Cinco Días.

[13] Fernandez, J. (2006). Gestión por competencias, un modelo estratégico para la dirección de Recursos Humanos. Madrid: Pearson Educación, S. A.

[14] García, O. (17 de Julio de 2010). SiOPE. org. Modelo para la gestión de competencias informacionales en las. Obtenido de Biblioteca Nacional de Cuba José Martí: http:// revistas.bnjm.cu/index.php/anales/article/ viewFile/40/32
[15] Giménez, A. V., \& Aymerich, M. I. (2016). Learning by competences (I). Identification of competences' profiles. Ensenanza de Las Ciencias, 34(3), 73-90. https://doi.org/10.5565/ rev/ensciencias. 1693

[16] Lawler, E. (1994). From Job - Based to Competence - Based Organizations. Journal of Organizational Behavior, 15(1), 3-15. https:// doi.org/10.1002/job.4030150103

[17] Lula, Paweł \& Oczkowska, Renata \& Kovaleva, Anna \& Wiśniewska, Sylwia. (2019). Methods of competency schema identification and evaluation. 13 Th IQC International Quality Conference, 18.

[18] Mc Clelland, D. C. (1973). Testing for competence rather than for "inteligence". American Psychcologist, 1-14.

[19] Mertens, L. (2000). La gestión por competencia laboral en la empresa y la Formación Basada en Competencias profesional. Madrid: Organización de Estados Iberoamericanos para la Educación, la Ciencia y la Cultura.

[20] Moreno, J., Pelayo, Y., \& Vargas, A. (Octubre de 2004). La gestión por competencias como herramienta para la dirección estratégica de recursos humanos en la sociedad del conocimiento. Revista de empresas(10).

[21] Navío,A. (2002). Lascompetencias delformador de Formación Basada en Competencias continuada. Análisis desde los programas de Formación Basada en Competencias de formadores. Barcelona, España: Octaedro.

[22] Noermijati, A. *, Achmad, S., \& Grace, M. (2019). The effect of knowledge management and the role of human resources for strategic planning and organizational performance: a study at hospitals of north sulawesi, indonesia. RJOAS, 12(96). https://doi.org/10.18551/ rjoas.2019-12.32

[23] Olaz, Á. (2009). Definición de un modelo de clima laboral basado en la gestión de competencias. Papers, revista de sociología(91).

[24] Rivero, E. J. R., Martín, A. E. R., \& Gil, D. N. (2015). Evidencia empírica de la adquisición de la competencia de resolución de problemas. Perfiles Educativos, 37(147), 50-66. https:// doi.org/10.1016/j.pe.2015.10.002

[25] Rocha, G. B., Passador, C. S., \& Shinyashiki, G. T. (2017). What is the social gain from competency management? The employees' perception at a Brazilian public university. 
Revista de Administração, 52(3), 233-245. https://doi.org/10.1016/j.rausp.2017.05.001

[26] Spencer, L. M., \& Spencer, S. M. (1993). Competencies at work: Models for superior performance. . New York: John Wiley \& Sons.

[27] Sotelo Medina, A. B. (2018). Impacto del soporte organizacional percibido y el compromiso organizacional en el balance vida-trabajo en la industria manufacturera de exportación grande enHermosillo, Sonora. RevistadeComunicación y Salud, 8(1), 45-59. https://doi.org/10.35669/ revistadecomunicacionysalud.2018.8(1).45-59
[28] Strukan, E., \& Nikolić, M. (2019). Impact of leadership on interpersonal trust at work in enterprises in bosnia and herzegovina) original scientific paper. journal of engineering management and competitiveness (jemc), 9(1), 48-59. http://www.tfzr.uns.ac.rs/jemc 